\title{
QUALITY OF LIFE OF CHRONIC HEPATITIS C PATIENTS RECEIVING SOVALDI TREATMENT REGIMENAT SHARQ ALMADINA IN ALEXANDERIA
}

Nagwa Fouad Shokeir, Heba Mahmoud Elweshahi, Nahla Ahmed Gamaleldin, Hend Hassan Abdelaziz Department of Community Medicine, Faculty of medicine, Alexanderia University, Egvpt.

\section{Introduction}

Hepatitis $\mathrm{C}$ virus $(\mathrm{HCV})$ infection is a worldwide public health problem affecting about $3 \%$ of the world's population . Nearly three quarters of infected individuals are living in middle income countries. China, Pakistan, Nigeria, Egypt, India, and Russia together accounted for more than half of total infections.

In response to the major problem of $\mathrm{HCV}$ in Egypt, the Egyptian Ministry of Health and Population (MOHP) launched the National Committee for Control of Viral Hepatitis. The introduction of sofosbuvir (Sovaldi) markedly changed therapeutic outcomes.

Health Related Quality of Life (HRQoL) has become a frequent and valuable outcome measure in clinical research with patients facing chronic diseases, including chronic liver diseases. The assessment of HRQoL in HCV patients under treatment might help to better understand ment of the patients.

\section{Aim of the work}

The study was conducted to assess quality of life of chronic hepatitis C patients receiving Sovaldi containing regimen at outpatient hepatology clinic in Sharq Al-madina hospital and identify factors associated with it.

\section{Subjects and methods}

A. Study Design:

A Cross sectional survey was conducted in order to achieve the study objectives.

Study setting: Outpatient Hepatology clinic Sharq Al-madina hospital in Alexandria, Egypt.

Study duration : The field work has started at beginning of April 2020 till the end November 2020 .

The target population: The target population was Chronic hepatitis $\mathrm{C}$ patients above 18 years of age of both sexes receiving Sovaldi treatment regimen for at least 3 months with no other chronic liver disease $(\mathrm{n}=165)$. The minimum sample size was estimated using PASS program $(\mathrm{n}=165)$. The minimum sample size was estimated using PASS program
version 20 using a two-sided one sample t- test at $\% 5$ level of significance and $85 \%$ power.
B.. Data collection methods and tools :

1. Data was collected from the studied $\mathrm{HCV}$ patients using face to face interview format which includes the following parts:

Part I: a structured interview format include personal and sociodemographic characteristics, history of associated co-morbid conditions, family history of hepatitis $\mathrm{C}$ affection, history of hepatitis $\mathrm{C}$ and detailed treatment history

Part II: The chronic liver disease questionnaire (CLDQ) to assess health related quality of life. A score was calculated for each domain, it was ranged from 1 (most impaired) to 7 , which indicates the minimal frequency of symptoms; indicating a favorable $\mathrm{HRQOL}$

Part III: The translated Arabic version of short form (SF 36) generic quality of life questionnaire. All questions were scored on a numeric scale using a scoring key. Then each item is scored on a 0 to 100 scale (transformed score). High atients to collect data about their laboratory findings before and after treatment.

\section{Results}

The vast majority of chronic HCV patients achieved sustained virological response after 12 weeks (SVR12).

Around two thirds of them reported improvement in their general health

Two fifths of all chronic HCV patients reported side effects after SOVALDI regimen, mostly headache, drowsiness, GIT disorders and fatigue.(figure 1 )

The median value of the overall SF-36Q score was 53.19 indicating a poor HRQoL. The highest scores were for role limitations due to emotional problems, physical and social functioning and lowest scores for energy/fatigue and emotional well-being.

The mean value of the average CLDQ score was $(4.59 \pm 1.28)$ reflecting fair HRQoL. The highest scores were for activity, systemic and abdominal symptoms domains. The lowest were for worry and fatigue.

Scores of almost all domains of SF-36Q were positively correlated with that of CLDQ

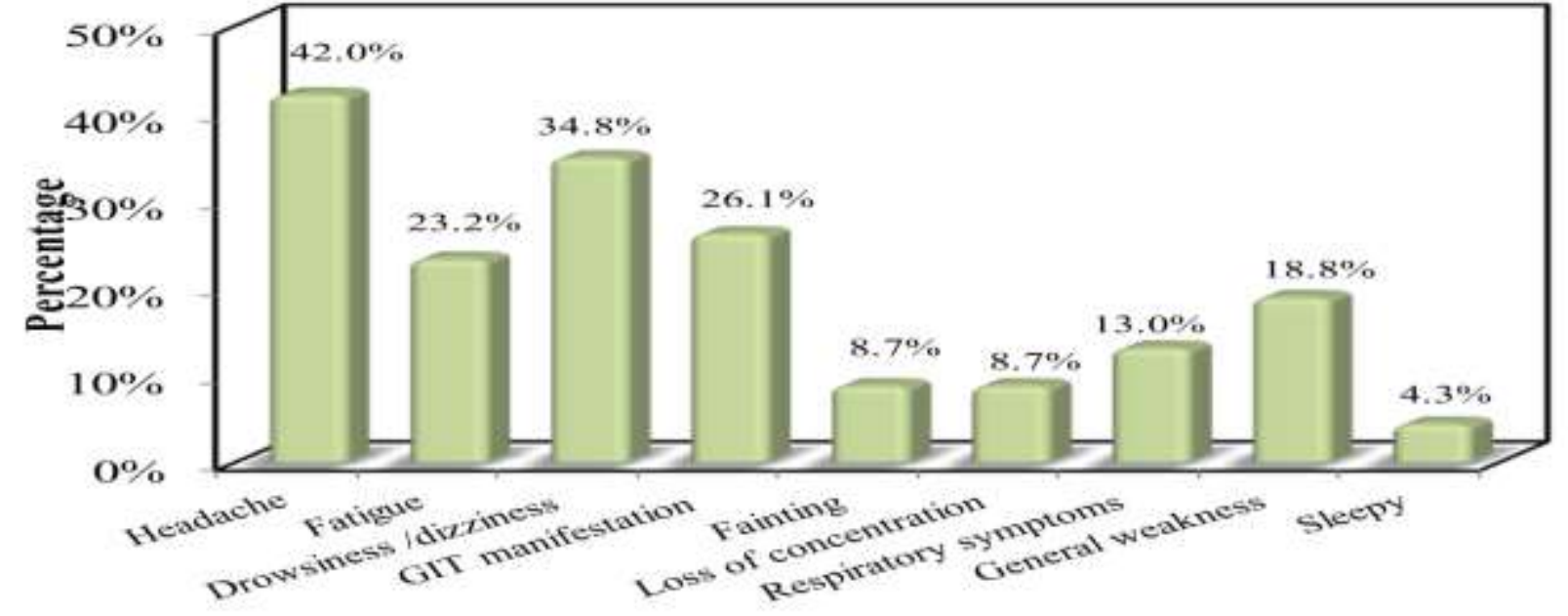

Figure 1: Distribution of the chronic HCV patients according to types of side effects associated with oral SOVALDI treatment regimen

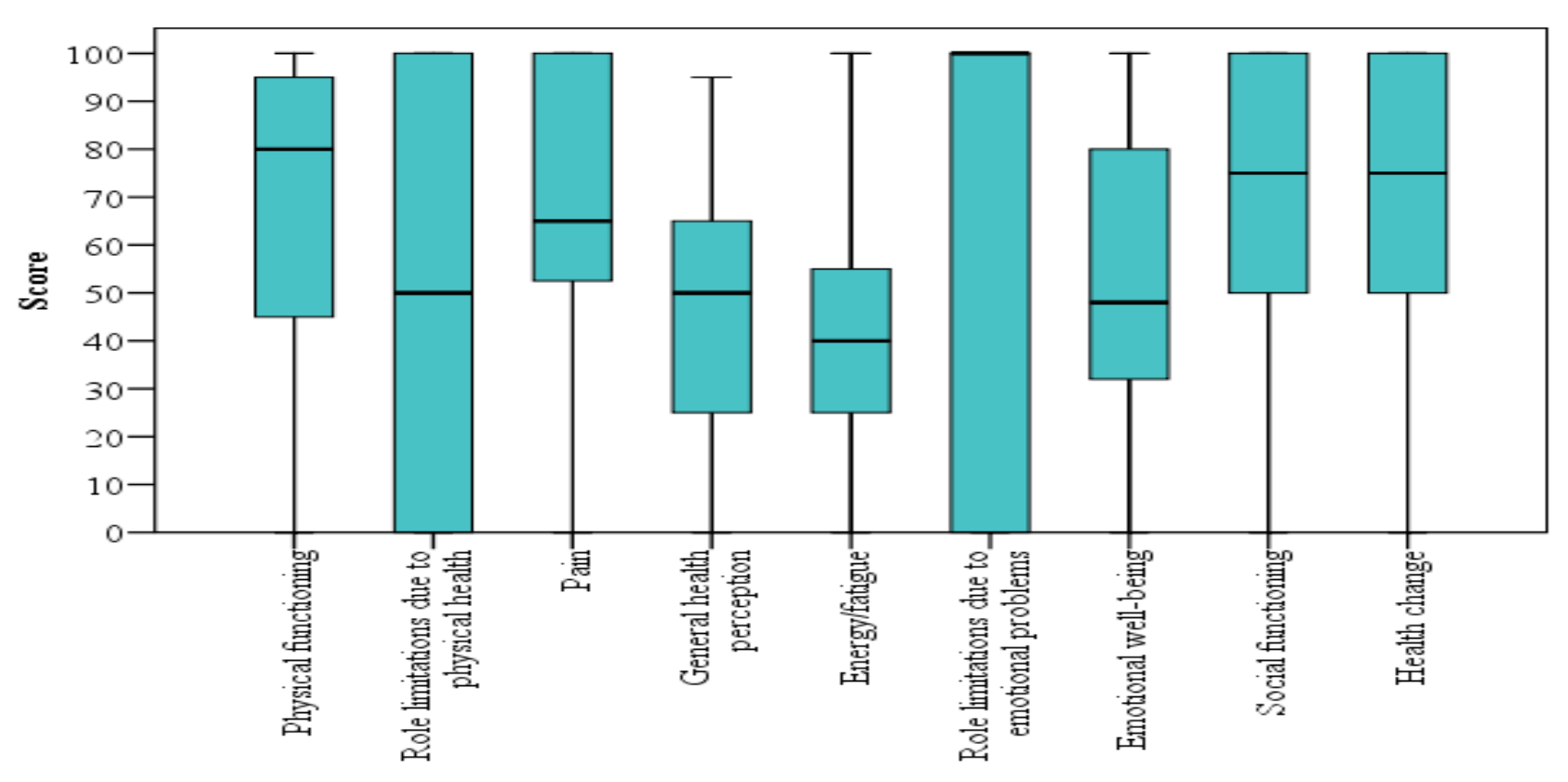

Conclusions

Although the majority of studied CHCV patients achieved SVR after 12 weeks from starting sovaldi treatment regimen, some patients exhibited inadequate HRQoL specially in the aspect of emotional well-being, fatigue and worry. HRQoL regular assessment for chronic $\mathrm{HCV}$ patients is essential for monitoring health outcomes while evaluating the management plan.

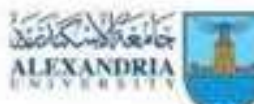
MEACULTY OP 1 |
$2021 @$ Alexandria Faculty of Medicine CC-BY-NC 